\title{
Lower expression of PDZRN3 induces endometrial carcinoma progression via the activation of canonical Wnt signaling
}

\author{
QIUHONG LI ${ }^{1}$, JIE ZHONG ${ }^{1}$, SHANGJIE YANG ${ }^{2}$ and YANPING LIANG ${ }^{1}$ \\ ${ }^{1}$ Department of Obstetrics and Gynecology, Shanghai Yangpu District Shidong Hospital, Shanghai 2000438;
${ }^{2}$ Department of Obstetrics and Gynecology, Yangpu Hospital Affiliated to Tongji University, Shanghai 200090, P.R. China
}

Received August 10, 2021; Accepted December 2, 2021

DOI: $10.3892 / \mathrm{ol} .2022 .13218$

\begin{abstract}
Endometrial carcinoma (EC) exhibits an extremely malignant biological behavior and has a high mortality rate. EC has recently become one of the most lethal cancers in women worldwide. E3 ubiquitin ligases play an important role in the biological function of healthy cells but can also contribute to tumorigenesis and cancer development. PDZ Domain Containing Ring Finger 3 (PDZRN3) is associated with cell differentiation and its structure includes the E3 ubiquitin ligase. However, the effects of PDZRN3 in EC remain unclear. Reverse transcription-quantitative PCR was used to detect the expression levels of PDZRN3 in EC cells, and the role of PDZRN3 in EC progression was determined using western blotting, MTT, colony formation, Transwell, subcutaneous tumor formation and pulmonary metastasis assays. A multi-pathway reporter arrays and western blotting were performed to investigate the potential biological mechanisms of PDZRN3 in EC. The present study demonstrated that PDZRN3 served an essential role in metastasis and proliferation of EC. PDZRN3 expression was lower in EC tissues compared with that in normal endometrial tissues. Low expression level of PDZRN3 in EC was correlated with certain clinicopathological features of patients with EC, such as the age of the patients, the tumor grade and the tumor subtype. The invasive and proliferative activities of EC cells with low expression of PDZRN3 were more potent than those of EC cells with high expression of PDZRN3, which was confirmed by in vivo and in vitro experiments. Furthermore, lower expression of PDZRN3 promoted metastasis and proliferation via activation of the canonical Wnt signaling pathway. The present study demonstrated that decreased PDZRN3 expression promoted metastasis and proliferation in EC cells via activation of the
\end{abstract}

Correspondence to: Dr Yanping Liang, Department of Obstetrics and Gynecology, Shanghai Yangpu District Shidong Hospital, 999 Shiguang Road, Shanghai 2000438, P.R. China

E-mail: ypl20170103@163.com

Key words: PDZ Domain Containing Ring Finger 3, endometrial carcinoma, proliferation, metastasis, Wnt signaling canonical Wnt signaling pathway, highlighting a potential biological therapeutic target for the management of EC.

\section{Introduction}

Endometrial cancer (EC) is one of the three main malignant tumors of the female reproductive system (1). Despite recent discoveries in the field of cancer therapy, no significant improvement has been made in the treatment of EC or its early detection. Most patients with EC exhibit tumor progression during their visits to their medical practitioners. ECs are usually spread locally or have metastasized (2-4). It has been shown that the degree of malignancy of EC is very high and is characterized by potent invasive and metastatic activities (5). Invasion and metastasis are the main obstacles to the long-term survival and quality of life of patients with EC $(5,6)$. These two processes are also considered as crucial biological behaviors of ECs, and may therefore be used as research directions for the treatment of Ec.

Ubiquitination is intricately involved in tumorigenesis and cancer development, including invasion and metastasis $(7,8)$. The E3 ubiquitination ligand is particularly important in tumorigenesis and cancer development (9-12). E3 ubiquitin ligases are a class of enzymes that are involved in the regulation of the conversion and activity of several target proteins. Significant differences are often noted in the structure and expression of several E3 ubiquitin ligases in tumor tissues compared with those of the corresponding normal tissues. In addition, these E3 ubiquitin ligases can have a significant impact on the regulation of malignant behavior, such as the regulation of epithelial-to-mesenchymal transition $(13,14)$. Increasing evidence has strongly suggested that the abnormal regulation of E3 ligases is related to the occurrence of cancer (12-14). Proteins with E3 ubiquitin ligases may be considered as potential cancer drug targets and prognostic biomarkers.

As a member of the Ligand of NUMB protein-X family, PDZ Domain Containing Ring Finger 3 (PDZRN3) contains the RING-type ubiquitin E3 ligase. The PDZRN3 gene is located in the $3 \mathrm{p} 13$ chromosomal region and is $\sim 242,568$ bases in length. The molecular mass of the corresponding protein is estimated to be $119,596 \mathrm{Da}$ and the protein is found in human and mouse tissues (15). Previous studies have indicated that PDZRN3 can induce the differentiation of 
$\mathrm{C} 2 \mathrm{C} 12$ mouse mesenchymal progenitor cells into myotubes or osteoblasts. This indicates that PDZRN3 exerts a certain effect on cell differentiation (16), which is also an extremely important biological function of tumor cells. Furthermore, the E3-ubiquitin ligase has been shown to affect the ubiquitination function of PDZRN3. The latter can promote the modification of the ubiquitination process of related molecules via the E3 ubiquitin ligase domain. This phenomenon is mediated by the decrease in Muscle-Specific Kinase expression via PDZRN3-induced ubiquitination $(17,18)$. PDZRN3 interacts with the E3 ubiquitin ligase domain. Cell differentiation provides a necessary theoretical basis for PDZRN3 in tumor invasion and metastasis, including EC.

The aim of the present study was to investigate the effects of PDZRN3 on EC cell proliferation, migration and invasiveness. Reverse transcription-quantitative PCR (RT-qPCR) was used to detect the expression levels of PDZRN3 in EC cells, and the role of PDZRN3 in EC progression was determined using western blotting, and MTT, colony formation, Transwell, subcutaneous tumor formation and pulmonary metastasis assays. A multi-pathway reporter array and western blotting were performed to investigate the potential biological mechanisms of PDZRN3 in EC.

\section{Materials and methods}

Cell culture and transfection. HEC-1-B and KLE cell lines were purchased from Tongpai (Shanghai) Biotechnology Co., Ltd. HEC-1-B cells were cultured in high glucose medium containing $10 \%$ FBS, $100 \mathrm{U} / \mathrm{ml}$ penicillin and $100 \mathrm{mg} / \mathrm{ml}$ streptomycin (all Hyclone; Cytiva). KLE cells were cultured in F12 medium (Hyclone; Cytiva) containing 10\% FBS, $100 \mathrm{U} / \mathrm{ml}$ penicillin and $100 \mathrm{mg} / \mathrm{ml}$ streptomycin. All cells were placed at $37^{\circ} \mathrm{C}$ in a humidified incubator containing $5 \% \mathrm{CO}_{2}$.

The designed plasmid (en-PDZRN3) was transfected into cells using Lipofectamine ${ }^{\mathrm{TM}} 3000$ (Invitrogen; Thermo Fisher Scientific, Inc). The plasmids were purchased from Wegene Biosciences Co., Ltd. The number of cells transduced with lentivirus was $\sim 2 \times 10^{5} /$ well. The next day, the original medium was replaced with $2 \mathrm{ml}$ fresh medium with $1 \mu \mathrm{l} / \mathrm{ml}$ Lipofectamine 3000 and adding $20 \mu \mathrm{l}$ virus suspension by incubating at $37^{\circ} \mathrm{C}$ for $24 \mathrm{~h}$. After $24 \mathrm{~h}$, the medium in the well was removed and $2 \mathrm{ml}$ of fresh medium ( $5 \mu \mathrm{g} / \mathrm{ml}$ puromycin) was added for $48 \mathrm{~h}$. The cells were amplified by changing the fresh medium, and the transfection efficiency was checked using RT-qPCR and western blotting. At $24 \mathrm{~h}$ before lentivirus transfection, the adherent cells were treated with $1 \times 10^{5} /$ well in a 24-well plate.

$R T-q P C R$. RNA was extracted from HEC-1-B/HEC-1B-PDZRN3 and KLE/KLE-PDZRN3 cells using TRIzol ${ }^{\circledR}$ (Invitrogen; Thermo Fisher Scientific, Inc.) and reverse transcribed into cDNA using a reverse transcription kit (Roche Applied Science) according to the manufacturer's instructions, followed by amplification using a qPCR kit (Roche Applied Science). The following thermocycling conditions were used for qPCR: $95^{\circ} \mathrm{C}$ for $10 \mathrm{~min}$; followed by 40 cycles of $95^{\circ} \mathrm{C}$ for $30 \mathrm{sec}, 60^{\circ} \mathrm{C}$ for $30 \mathrm{sec}$ and $72^{\circ} \mathrm{C}$ for $30 \mathrm{sec}$, and final extension at $72^{\circ} \mathrm{C}$ for $2 \mathrm{~min}$. The expression level of PDZRN3 was normalized to endogenous control GAPDH and expressed using the $2^{-\Delta \Delta C q}$ method (19). Each assay was repeated three times. The sequences of the primers were as follows: GAPDH forward, 5'-CACCGCAAATGCTTCTAGGC-3' and reverse, 5'-GATCTCCACACACCTGCACT-3'; and PDZRN3 forward, 5'-ATTATTGAGGTCAACGGCAG-3' and reverse, 5'-AGG GCCATGATATGTTCAAAG-3'.

Western blotting. Cells were lysed using RIPA lysis buffer (New Cell \& Molecular Biotech Co., Ltd.). Following estimation of the protein concentration using a BCA kit (Beyotime Institute of Biotechnology), proteins $(40 \mu \mathrm{g})$ were separated by $12 \%$ SDS-PAGE and transferred onto a PVDF membrane (MilliporeSigma). Membranes were incubated with primary antibodies against PDZRN3 $(1: 2,000$; cat. no. Ag24455; ProteinTech Group, Inc.), $\beta$-catenin (1:2,000; cat. no. 17565-1-AP; ProteinTech Group, Inc.) and tubulin (1:2,000; cat. no. 11224-1-AP; ProteinTech Group, Inc.) at $4^{\circ} \mathrm{C}$ overnight. Membranes were washed by 1X PBST solution and incubated with the corresponding secondary antibodies (HRP-conjugated Affinipure goat anti-rabbit $\operatorname{IgG}(\mathrm{H}+\mathrm{L})$; 1:5,000; cat. no. SA00001-2; ProteinTech Group, Inc.). Enhanced chemiluminescence reagent (ebiogen, Inc.) was used to detect the signal on the membrane. The data were analyzed via densitometry and normalized to expression of the internal control (tubulin) by ImageJ 1.8. (National Institutes of Health).

MTT assay. Cells $\left(1 \times 10^{4}\right)$ in the HEC-1-B/HEC-1-B-PDZRN3 and KLE/KLE-PDZRN3 groups were incubated into 96-well plates and cultured for 7 days. A total of $20 \mu \mathrm{l}$ MTT $(5 \mathrm{mg} / \mathrm{ml})$ was added into the culture medium and incubated for $4 \mathrm{~h}$. Subsequently, $200 \mu 1$ DMSO was added following removal of the medium to dissolve the MTT formazan. The absorbance was read at $490 \mathrm{~nm}$ on a microplate reader.

Colony formation assay. Colony formation assay was performed to evaluate the proliferative ability of EC cells. A total of 500 EC cells derived from the HEC-1-B/HEC-1-B-PDZRN3 and KLE/KLE-PDZRN3 groups were seeded into six-well plates and stained at room temperature for $10 \mathrm{~min}$ with $0.1 \%$ crystal violet following 14 days of incubation. The number of colonies (cell clusters with a diameter $>1 \mathrm{~mm}$ ) was counted manually using a microscope.

Invasion assay. A total of $5 \times 10^{5}$ cells were resuspended in $200 \mu \mathrm{l}$ serum-free medium and subsequently seeded to the upper chamber of a Transwell (Corning, Inc.) insert precoated with $1 \mu \mathrm{g} / \mu \mathrm{l}$ Matrigel (BD Biosciences). Complete medium was added to the lower chamber to stimulate cell invasion. Following $48 \mathrm{~h}$ of cell culture, the cells that did not cross the membrane were wiped with a cotton swab, while the cells adhering to the lower surface of the membrane were stained with $0.1 \%$ crystal violet solution at room temperature for $10 \mathrm{~min}$. The number of invading cells was counted in five randomly selected fields using a light microscope (magnification, x200; Olympus Corporation). All experiments were performed three times.

Migration assay. A total of $5 \times 10^{5}$ cells were resuspended in $200 \mu 1$ serum-free medium and subsequently seeded to the upper chamber of a Transwell insert without Matrigel. 

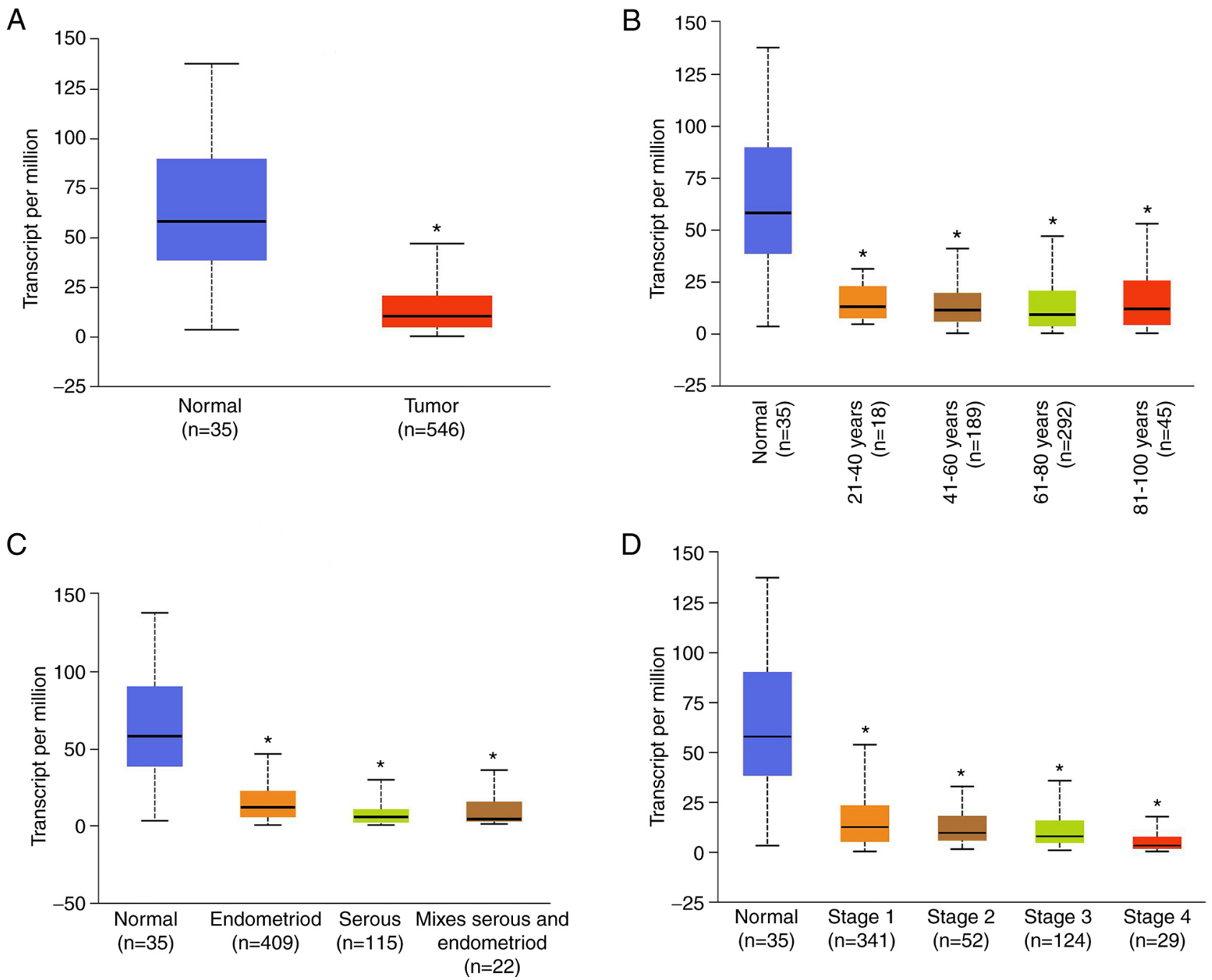

Figure 1. PDZRN3 expression is significantly decreased in EC tissues. (A) PDZRN3 mRNA expression was analyzed by RT-qPCR in human EC tissues in TCGA database. (B) PDZRN3 mRNA expression was analyzed by RT-qPCR in human EC tissues in TCGA database. (C) PDZRN3 mRNA expression was analyzed by RT-qPCR in human EC tissues in TCGA database. (D) PDZRN3 mRNA expression was analyzed by RT-qPCR in human EC tissues in TCGA database. " $\mathrm{P}<0.05$. RT-qPCR, reverse transcription quantitative PCR; PDZRN3, PDZ Domain Containing Ring Finger 3; TCGA, The Cancer Genome Atlas; EC, endometrial carcinoma.

All other procedures were performed as described for the invasion assay.

Subcutaneous tumor formation assay. A total of 40 BALB/ c-nude female mice aged 6-8 weeks were purchased from the Slk Jing Da Laboratory Animal Co., Ltd. and the experiments were performed in a SPF-level sterile laboratory at the Animal Testing Center of Tongji University. All experimental procedures were approved by the Animal Ethics Committee of Shanghai Yangpu District Shidong Hospital. All mice were $\sim 20 \mathrm{~g}$ in weight when purchased. Mice were housed with free access to food and water in a temperature- and light-regulated pathogen-free room (temperature, $24 \pm 1^{\circ} \mathrm{C}$; humidity, $60 \pm 5 \%$; 12-h light/dark cycle). In the present study, the maximum length and diameter of the tumor was $<15 \mathrm{~mm}$ and the volume and weight of the tumor did not exceed $20 \%$ of body weight. The tumor load of each mouse was no more than 2 tumor lesions. Direct cervical dislocation was used for euthanasia, which provides a rapid, painless and stress-free death. All mice were judged dead following no response to stimulation, no chest fluctuation, no heartbeat and significant decrease in body temperature. After death, all mice were treated with humane care and pollution-free treatment in the animal experiment center. In the animal center, the animal keeper checks once a day to ensure the health of mice. There were 5 nude mice in the HEC-1-B/HEC-1-B-PDZRN3 and KLE/KLE-PDZRN3 groups. The HEC-1-B/HEC-1-B-PDZRN3 cells and KLE/KLE-PDZRN3 cells $\left(5 \times 10^{6}\right)$ for each group were injected subcutaneously into nude mice (7-8 weeks, female, BALB/c) to assess their tumorigenic capacity. The tumor samples were collected following 4 weeks of incubation and after euthanasia. Tumor volume was calculated as follows: Tumor volume $\left(\mathrm{mm}^{3}\right)=(\mathrm{AxB} 2) / 2$, where A corresponds to length and $\mathrm{B}$ to width. The in vivo experiments lasted for 4 weeks, during which the animals suffered no pain or showed no sign of distress, such as rapid weight loss (over 20\%), loss of appetite, weakness, organ and tissue infection or tumor overweight.

Pulmonary metastasis assay. The concentration of the HEC-1-B/HEC-1-B-PDZRN3 cells and KLE/KLE-PDZRN3 


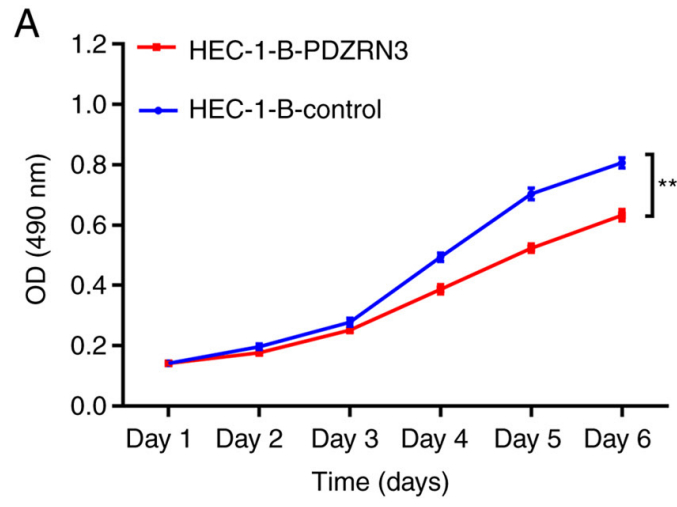

B HEC-1-B-control

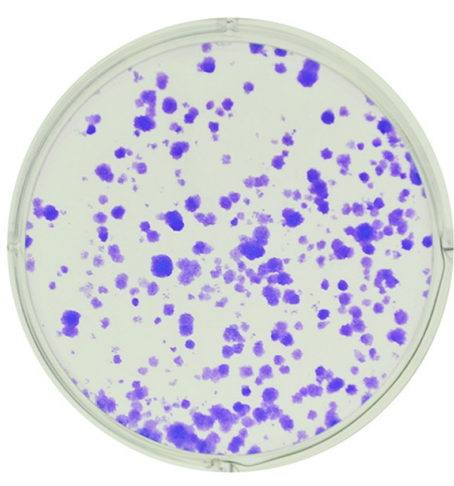

KLE-control

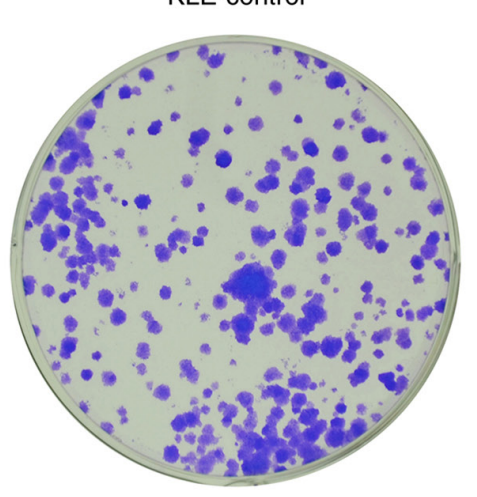

HEC-1-B-PDZRN3

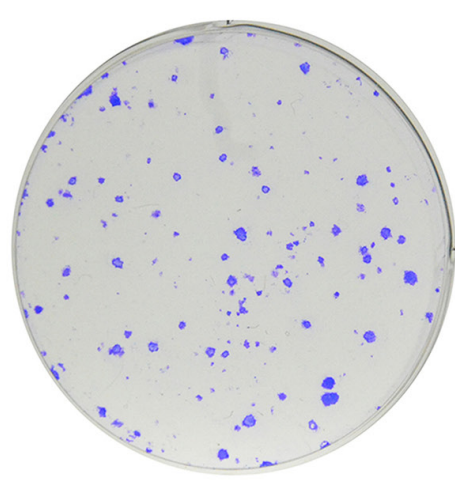

KLE-PDZRN3

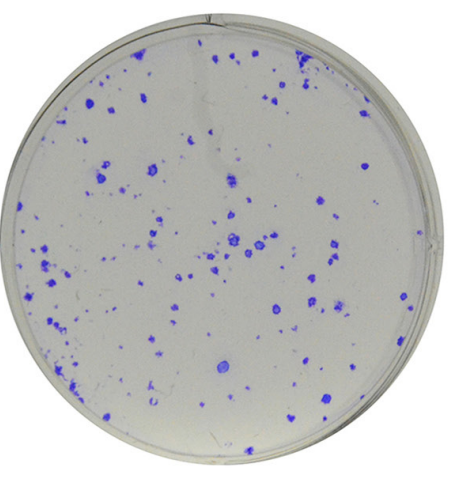

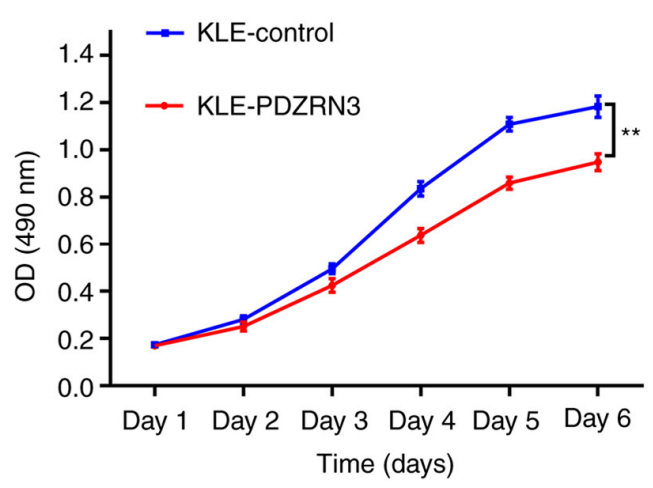

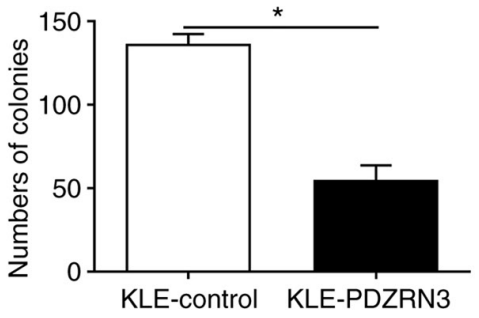

Figure 2. PDZRN3 overexpression inhibits endometrial carcinoma cells proliferative ability in vitro. (A) MTT assay was performed to evaluate the proliferation of HEC-1-B and KLE cells following PDZRN3 overexpression. (B) Colony formation assay was performed to determine the proliferative ability of HEC-1-B and KLE cells following overexpress-PDZRN3 transfection and control groups. ${ }^{*} \mathrm{P}<0.05$ and ${ }^{* *} \mathrm{P}<0.01$. PDZRN3, PDZ Domain Containing Ring Finger 3; OD, optical density.

cells was adjusted to $5 \times 10^{7}$ cells $/ \mathrm{ml}$ using a cell counter. Each nude mouse was injected with $100 \mu \mathrm{l}$ cell suspension twice a week. Following 4 weeks of incubation, the nude mice were sacrificed by cervical dislocation. The lungs of the nude mice were completely removed and subsequently immersed in $10 \%$ formalin solution. The lungs of the nude mice were embedded in paraffin and sectioned into $\sim 5 \mu \mathrm{m}$ thick slices. Following hematoxylin and eosin staining, the metastasis of the lung tumors was observed by microscopy and the number of metastatic colonies was counted.

Hematoxylin and eosin $(H \& E)$ staining and immunohistochemical (IHC) staining. For H\&E staining, the slides were incubated at $62^{\circ} \mathrm{C}$ for $1 \mathrm{~h}$. The slices were dewaxed in xylene solution and rehydrated with graded alcohol $(100,95$, 75 and 50\%) and distilled water. The slides were stained with hematoxylin solution at room temperature for $5 \mathrm{~min}$, then immersed in $1 \%$ acid ethanol at room temperature for $5 \mathrm{~min}$, prior to washing with distilled water. The slides were stained with eosin solution for $3 \mathrm{~min}$, then washed with graded alcohol $(50,75,95$ and $100 \%)$ for dehydration. The slides were then dried with xylene solution at room temperature. The H\&E images were captured at x100 magnification using a microscope camera system (Olympus Corporation).

For IHC staining, the slides were dewaxed in xylene solution and rehydrated in graded alcohol (100, 95, 75 and 50\%) and distilled water, then treated with $3 \%$ methanol hydrogen peroxide at room temperature for $30 \mathrm{~min}$. After washing 
with PBS three times, they were heated in a microwave oven with $10 \%$ citrate buffer for 10 min twice and cooled to room temperature, then $10 \%$ BSA was added for $30 \mathrm{~min}$. The slices were incubated with anti-Ki-67 antibody $(1: 2,000$; cat. no. 27309-1-AP; ProteinTech Group, Inc.) overnight at $4^{\circ} \mathrm{C}$. The next day, after washing with PBS, the sections were incubated with HRP-conjugated secondary antibody at room temperature for $1 \mathrm{~h}$. DAB solution was used for staining for $5 \mathrm{~min}$ and hematoxylin was used for re-staining the nucleus. The slides were then washed with graded alcohol $(50,75,95$ and $100 \%$ ) for dehydration, then dried with xylene solution at room temperature. The images were captured at x400 magnification using a microscope camera system (Olympus Corporation).

Multi-pathway reporter array. A Signal Finder 10-Pathway Reporter Array (SA Biosciences) was used for the signaling pathway assessment using the reverse transfection technique. The relative firefly luciferase activity of the HEC-1-B/HEC-1-B-PDZRN3 and KLE /KLE-PDZRN3 groups was calculated using a dual luciferase reporter and analysis system (Promega Corporation) and normalized to that of Renilla luciferase.

Statistical analysis. All data are expressed as the mean \pm standard deviation. SPSS 18.0 (SPSS, Inc.) and GraphPad Prism 8 (GraphPad Software,Inc.) were used for data analysis. Statistical comparisons of the data were performed using unpaired t-test between PDZRN3-treated cell groups/cancer tissues and their control cell groups. Associations between PDZRN3 expression and clinicopathological characteristics were analyzed using Pearson's $\chi^{2}$ test. One-way (or two-way ANOVA) was used for multiple comparisons of different cell groups followed by Bonferroni's correction, Benjamini-Hochberg method or Dunnett's test. All experiments were performed at least three times. $\mathrm{P}<0.05$ was considered to indicate a statistically significant difference.

\section{Results}

PDZRN3 expression is significantly lower in EC tissues. The expression level of PDZRN3 in EC was examined using the public database The Cancer Genome Atlas (TCGA). The results demonstrated that PDZRN3 expression was significantly lower in EC tissues compared with normal tissues ( $\mathrm{P}<0.001$; Fig. 1A). In addition, PDZRN3 expression was associated with the age of the patients, the tumor grade and the tumor subtype (Fig. 1). Different age groups were selected from the TCGA database as follows: $21-40,41-60,61-80$ and 81-100 years. The expression level of PDZRN3 in patients with EC was significantly lower than those in normal endometrial tissues $(\mathrm{P}<0.001$; Fig. 1B). Furthermore, the expression level of PDZRN3 in EC of different pathological classification types (endometrioid, serous or mixed) was significantly lower than those in normal endometrial tissues ( $\mathrm{P}<0.001$; Fig. $1 \mathrm{C})$. In addition, the clinical stages of EC were also identified as follows: Stage 1, stage 2, stage 3 and stage 4 . The data further demonstrated that the expression level of PDZRN3 in EC tissues was significantly decreased $(\mathrm{P}<0.001$; Fig. 1D) compared with normal group. These results suggested that lower PDZRN3 expression in

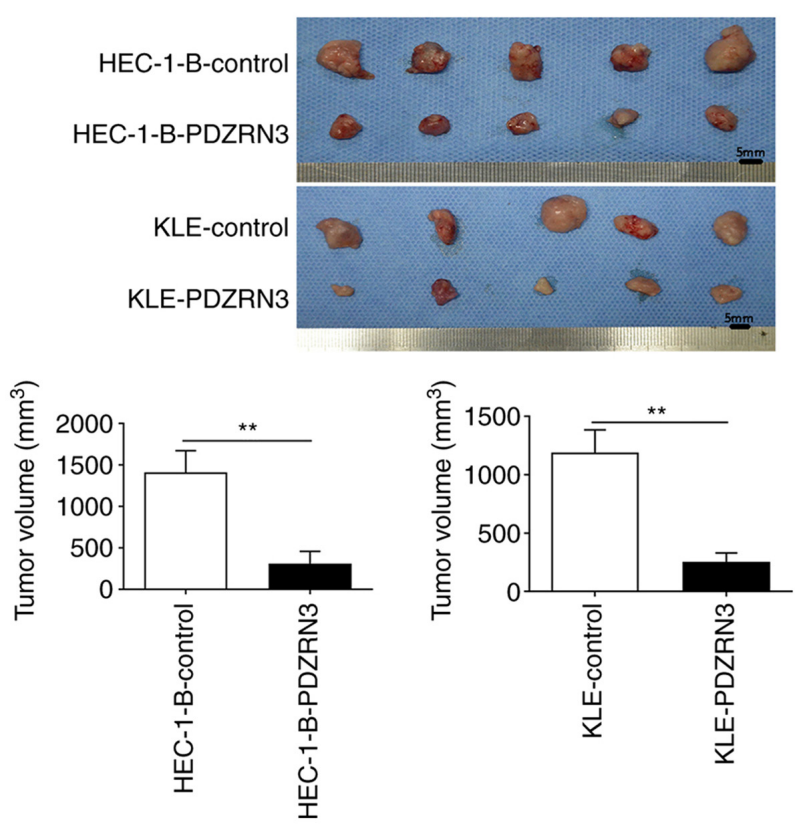

Figure 3. PDZRN3 overexpression inhibits proliferation ability of EC cells in vivo. Subcutaneous tumor formation assay was performed to show that PDZRN3 overexpression inhibited EC tumor growth in vivo. ${ }^{* *} \mathrm{P}<0.01$. PDZRN3, PDZ Domain Containing Ring Finger 3; EC, endometrial carcinoma.

ECs may therefore be correlated with EC progression and accelerate invasion and metastasis of EC.

PDZRN3 promotes EC cell proliferation. To evaluate the function of PDZRN3 in EC, PDZRN3 was overexpressed using a lentiviral vector. Ectopic PDZRN3 was constitutively expressed in HEC-1-B cells (HEC-1-B-PDZRN3) and KLE cells (KLE-PDZRN3). The expression levels of PDZRN3 were assessed by RT-qPCR and western blotting (Fig. S1). Furthermore, HEC-1-B-PDZRN3 and KLE-PDZRN3 cells exhibited lower absorbance compared with HEC-1-B- and KLE-control cells as determined by the MTT assay, which indicated a decreased proliferative rate (Fig. 2A). In addition, the high PDZRN3 expression cell groups HEC-1-B-PDZRN3 and KLE-PDZRN3 formed a lower number of colonies according to results from the colony formation assay (Fig. 2B) compared with the control-PDZRN3 cell groups HEC-1-B- and KLE-control cells. To verify these findings in vivo, subcutaneous xenograft tumor models were established. Following 4 weeks of incubation, the HEC-1-B-PDZRN3 and KLE-PDZRN3 cell-derived tumors at the subcutaneous implantation sites were significantly smaller and grew more slowly than those from the HEC-1-Band KLE-control groups (Fig. 3). In addition, Ki-67 staining for these tumors confirmed that high PDZRN3 expression groups HEC-1-B-PDZRN3 and KLE-PDZRN3 had fewer Ki-67 positive cells (Fig. S2) compared with the control-PDZRN3 groups HEC-1-B- and KLE-control cells. Taken together, these results suggested that decreased PDZRN3 expression could promote EC cell proliferation.

Lower PDZRN3 promotes EC cell migration and invasion. Transwell invasion and migration assays were used to investigate the invasive and migratory abilities of the cells. 
A

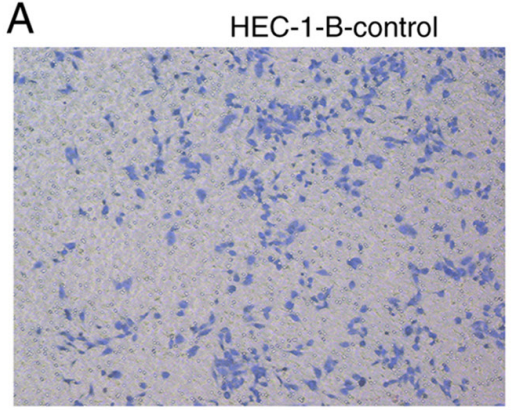

KLE-control

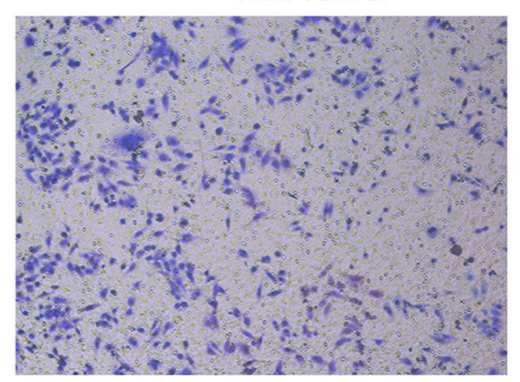

B

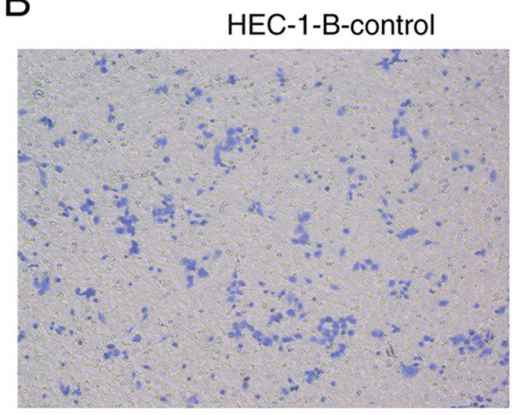

KLE-control

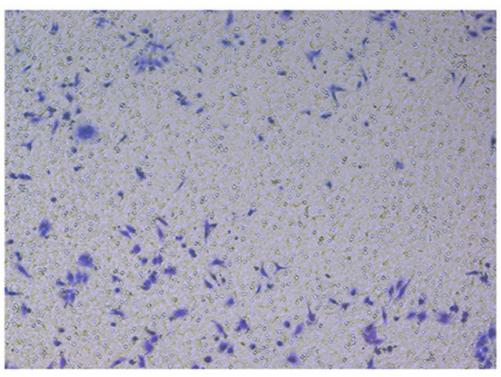

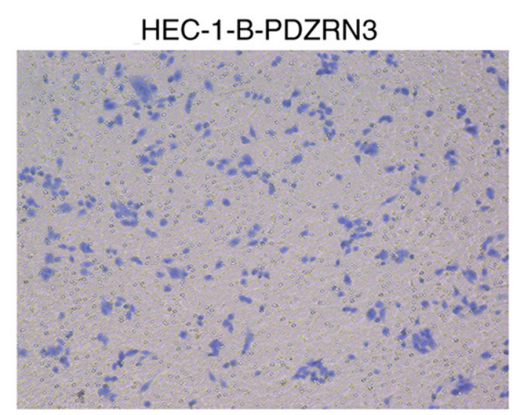

KLE-PDZRN3

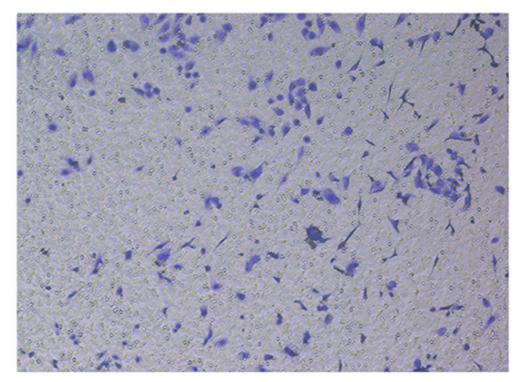

HEC-1-B-PDZRN3

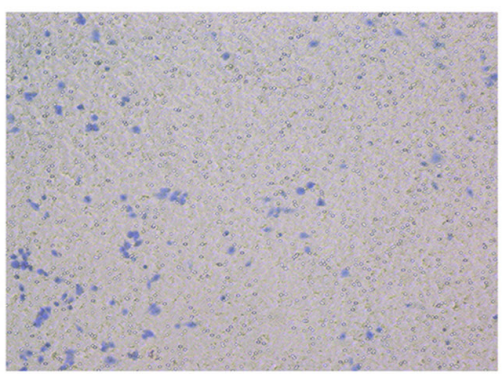

KLE-PDZRN3

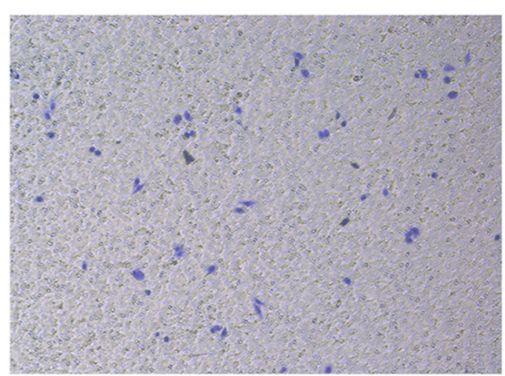

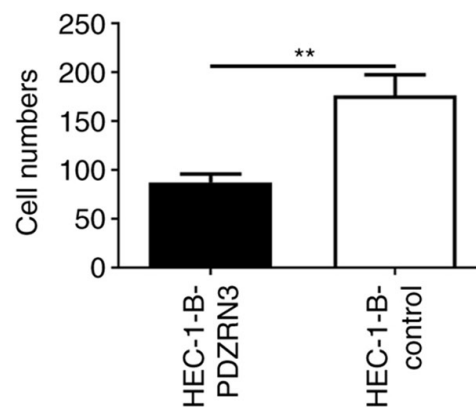

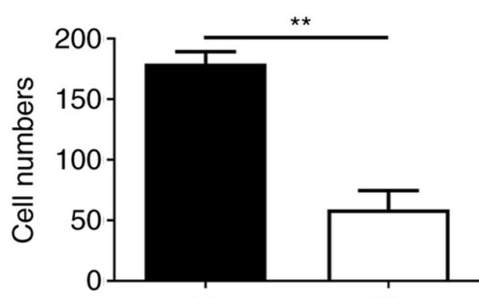

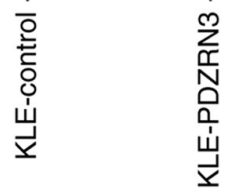
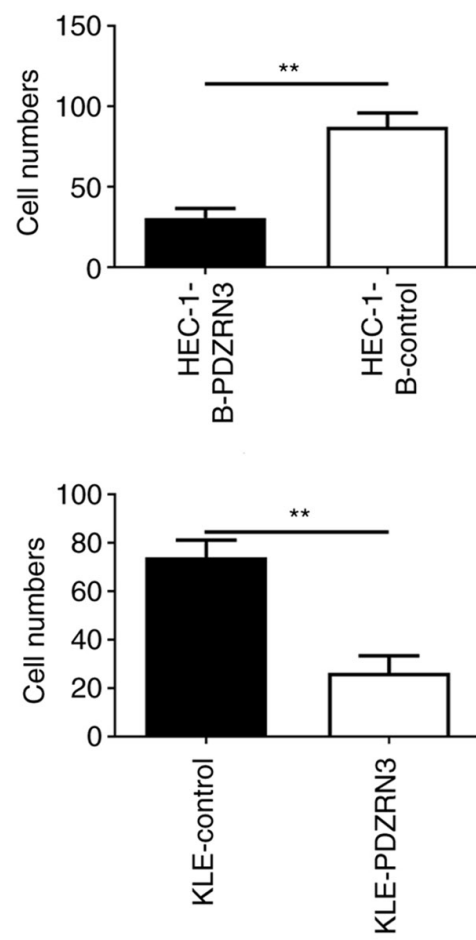

Figure 4. PDZRN3 overexpression inhibits the invasion ability of endometrial carcinoma cells in vitro. (A) Transwell migration experiment was performed to evaluate the migratory ability of HEC-1-B and KLE cells following PDZRN3 overexpression. (B) Transwell invasion experiment was performed to determine the invasive ability of HEC-1-B and KLE cells following PDZRN3 overexpression. ${ }^{* *} \mathrm{P}<0.01$. PDZRN3, PDZ Domain Containing Ring Finger 3.

The results indicated that HEC-1-B-PDZRN3 cells exhibited significantly reduced migratory ability compared with the HEC-1-B-control cells. Similarly, KLE- PDZRN3 cells exhibited significantly lower migratory ability compared with the KLE-control cells (Fig. 4A). The results from the invasion assays indicated that HEC-1-B-PDZRN3 cells exhibited a significantly lower number of invading cells compared with the HEC-1-B-control cells. In addition, KLE-PDZRN3 cells exhibited a significantly lower number of invading cells compared with the KLE-control cells (Fig. 4B). Furthermore, a pulmonary metastasis nude mouse model was established and the invasive activity was assessed by immunohistochemical analysis. The results indicated that the number of lung metastases in tumors of mice injected with cells overexpressing PDZRN3 (HEC-1-B-PDZRN3 and KLE-PDZRN3) was significantly lower than that in the control-PDZRN3 cell group (HEC-1-B- and KLE-control cells; Fig. 5). Taken together, these results indicated that lower expression of PDZRN3 may promote the metastasis of EC cells via increased proliferation, migration and invasion of EC cells. 

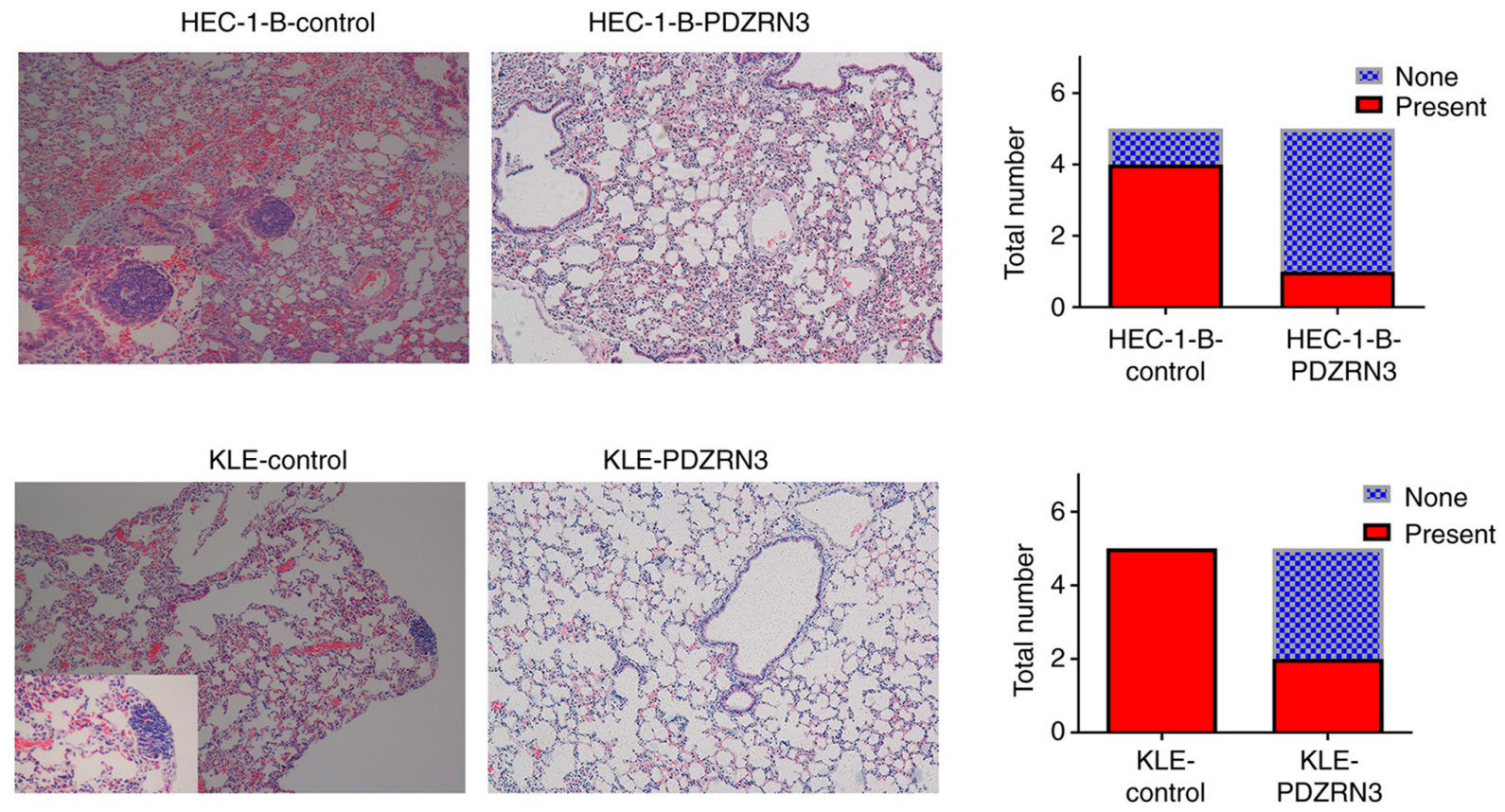

Figure 5. PDZRN3 overexpression inhibits the invasive ability of EC cells in vivo. Hematoxylin and eosin staining showed metastasis in lung tissues (left panels). Pulmonary metastasis lesions advanced in groups of lower PDZRN3 expression (right panels). Magnification, x100.

PDZRN3 modulates EC progression via the canonical Wnt signaling pathway. PDZRN3 expression is associated with canonical/non-canonical Wnt signaling in non-tumor cell differentiation microenvironment (20). To systemically screen the potential signaling molecules that could interact with PDZRN3, a Signal Finder Cancer 10-Pathway Reporter Array was assessed by RT-qPCR analysis. The results demonstrated that PDZRN3 significantly attenuated the activity of Wnt signaling in the high PDZRN3 expression groups HEC-1-B-PDZRN3 and KLE-PDZRN3, whereas it increased Wnt signaling activity in the control-PDZRN3 groups HEC-1-B-control and KLE-control cells (Fig. 6A). Furthermore, $\beta$-catenin expression level was assessed in these cell groups and the data demonstrated that ectopic expression of PDZRN3 in HEC-1-B-PDZRN3 and KLE-PDZRN3 groups was accompanied with decreased $\beta$-catenin accumulation (Fig. 6B). Conversely to these observations, decreased PDZRN3 expression and increased $\beta$-catenin accumulation were observed in HEC-1-B- and KLE-control groups (Fig. 6B). These results indicated that PDZRN3 could modulate the canonical Wnt signaling pathway.

\section{Discussion}

In clinical, it is found that EC has high malignancy and low survival rate. Tumor tissues exhibit uncontrolled proliferation compared with normal tissues (21). Tumor invasion and metastasis are often a direct cause of poor prognosis and high mortality rates in patients with tumors, including patients with EC $(22,23)$. In addition, it is recognized that ubiquitination system serves a crucial role in tumor genesis and development, including invasion and metastasis. E3 ubiquitination ligands have been reported to have important clinical significance in the regulation of cell motility and of tumor invasion and metastasis (24,25). Molecules with such structures and functions may therefore play a crucial role in tumor invasion and metastasis and may have potential value in clinical research. Based on this evidence, the assessment of the structure and function of PDZRN3 may be of considerable interest.

Previous studies reported that PDZRN3 and its E3 ubiquitin ligase domain serve an important role in ubiquitination function and in promoting cell differentiation $(26,27)$, which confirms the hypothesis that it may be involved in promoting tumor progression. Following analysis of the public database TCGA, significant differences in PDZRN3 expression were identified in EC tissues. PDZRN3 expression was associated with the age of the patients, tumor grade and tumor subtype. In addition, PDZRN3 expression was associated with certain EC subtypes, suggesting that PDZRN3 may be used to distinguish the clinical subtypes of EC. In summary, this study demonstrated that PDZRN3 may have ubiquitination function, and by checking the public database TCGA, we found that PDZRN3 was significantly lower in EC compared with normal tissues. The present study was incomplete since it lacked clinical case data and because it failed to collect comprehensive data relevant to the mechanism of action of PDZRN3.

The present study primarily focused on assessing the invasive, metastatic and proliferative functions of EC with regard to PDZRN3 expression. The results derived from the in vivo and in vitro experiments indicated that low expression of PDZRN3 could affect these characteristics of EC cells. One limitation of the present study is that the impact of PDZRN3 on other tumor cell characteristics, such as apoptosis and immune escape, was not examined. Furthermore, the effect of PDZRN3 on other biological behaviors could not be comprehensively evaluated. In addition, it has been reported that PDZRN3 can promote the differentiation of mesenchymal progenitor cells into myotubes in 2C12 mice, which indicates that PDZRN3 could promote the transformation from poorly differentiated to highly differentiated cells $(16,28)$. Tumor cells are mainly poorly differentiated 
A

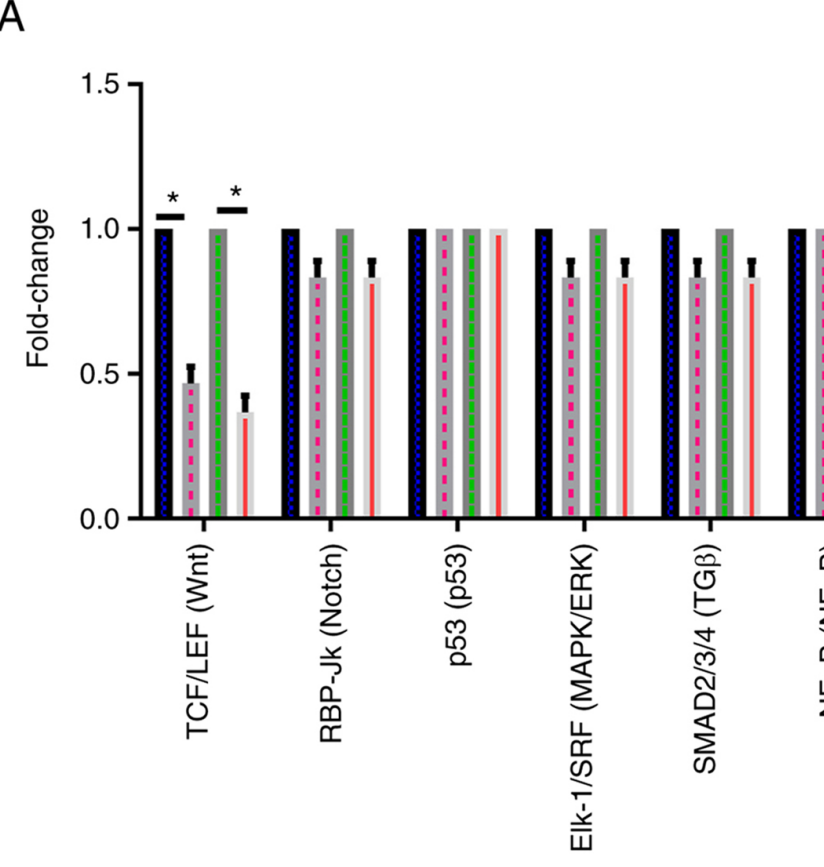

HEC-1-B-control

Bad HEC-1-B-PDZRN3

KLE-control

IIII KLE-PDZRN3

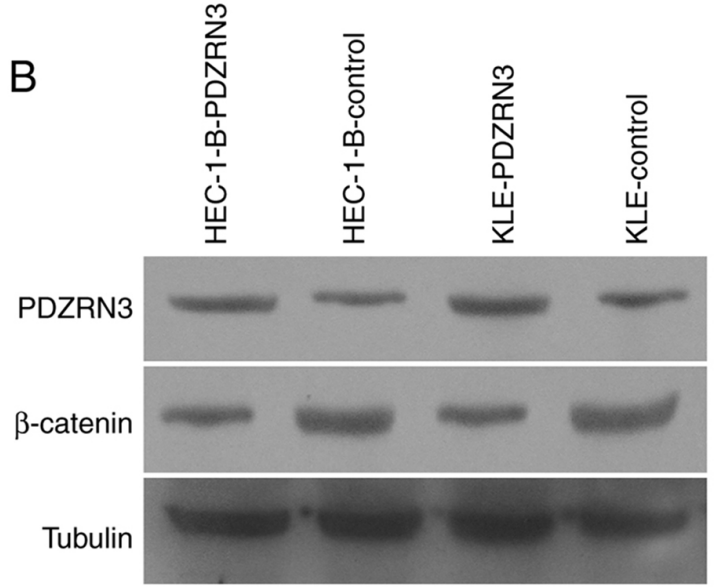

Figure 6. PDZRN3 modulates EC progress through canonical Wnt signaling. (A) Cignal Finder Cancer 10-Pathway Reporter Array showed the signaling changes in PDZRN3 different expression cells. (B) PDZRN3 and $\beta$-catenin protein expression determined by western blot in EC cells following PDZRN3 overexpression. " $\mathrm{P}<0.05$. PDZRN3, PDZ Domain Containing Ring Finger 3; EC, endometrial cancer.

cells. Whether the expression of PDZRN3 could induce the differentiation of tumor cells is also worthy of investigation. In addition, due to the limitation of research funds, the role of PDZRN3 ubiquitination in tumors was not examined, and should thus be investigated in future studies.

From preliminary screening, it was found that PDZRN3 was related to the activation of the canonical Wnt signaling pathway. It is well established that the Wnt signaling pathway is closely related to specific tumor biological characteristics $(29,30)$, such as proliferation or invasion, and the PDZRN3-induced biological behavior is likely to require the activation of the canonical Wnt signaling pathway (16). Additional investigation is required to confirm the important role of the canonical Wnt signaling pathway in EC to reveal its downstream target and determine whether this target is ubiquitinated by the E3 ubiquitinated ligand. The specific downstream targets and related functional verification of the canonical Wnt signaling pathway were not investigated in the present study.

In summary, the present study indicated that PDZRN3 inhibited the invasion, metastasis and proliferation of EC cells. By using preliminary signaling pathway screening, PDZRN3 was identified as a potential target responsible for the biological behavior of EC via activation of the Wnt signaling pathway. PDZRN3 expression may therefore provide important clinical guidance for predicting the survival of patients with $\mathrm{EC}$ and for the development of targeted therapeutics for EC. 


\section{Acknowledgements}

Not applicable.

\section{Funding}

No funding was received.

\section{Availability of data and materials}

The datasets used and/or analyzed during the current study are available from the corresponding author on reasonable request.

\section{Authors' contributions}

YL designed the project and funded all the experiments. QL completed all the experiments. JZ and SY were involved in drafting the manuscript, revising it critically for important intellectual content and assisted with the experiments. YL, QL, JZ and SY confirm the authenticity of all the raw data. All authors read and approved the final manuscript.

\section{Ethics approval and consent to participate}

Animal experimental procedures were approved by the Animal Ethics Committee of Shanghai Yangpu District Shidong Hospital.

\section{Patient consent for publication}

Not applicable.

\section{Competing interests}

The authors declare that they have no competing interests.

\section{References}

1. Bray F, Ferlay J, Soerjomataram I, Siegel RL, Torre LA and Jemal A: Global cancer statistics 2018: GLOBOCAN estimates of incidence and mortality worldwide for 36 cancers in 185 countries. CA Cancer J Clin 68: 394-424, 2018.

2. Washington CR, Haggerty A, Ronner W, Neff PM and Ko EM: Knowledge of endometrial cancer risk factors in a general gynecologic population. Gynecol Oncol 158: 137-142, 2020.

3. Byrne FL, Martin AR, Kosasih M, Caruana BT and Farrell R: The role of hyperglycemia in endometrial cancer pathogenesis. Cancers (Basel) 12: 1191, 2020.

4. Nagao S: IV.Endometrial Cancer. Gan To Kagaku Ryoho 47: 247-251, 2020 (In Japanese).

5. Lamothe S and Ramia de Cap M: Survival analysis and treatment effects in patients with endometrial cancer and POLE mutations. Cancer 127: 4306-4307, 2021.

6. Luna C, Balcacer P, Castillo P, Huang M and Alessandrino F: Endometrial cancer from early to advanced-stage disease: An update for radiologists. Abdom Radiol (NY) 46: 5325-5336, 2021.

7. Deng L, Meng T, Chen L, Wei W and Wang P: The role of ubiquitination in tumorigenesis and targeted drug discovery. Signal Transduct Target Ther 5: 11, 2020.

8. Pérez-Benavente $\mathrm{B}$, Nasresfahani $\mathrm{AF}$ and Farràs R: Ubiquitinregulated cell proliferation and cancer. Adv Exp Med Biol 1233 $3-28,2020$
9. Faktor J, Pjechová M, Hernychová L and Vojtěšek B: Protein ubiquitination research in oncology. Klin Onkol 32 (Suppl 3): S56-S64, 2019.

10. Fan Q, Wang Q, Cai R, Yuan $\mathrm{H}$ and Xu M: The ubiquitin system: orchestrating cellular signals in non-small-cell lung cancer. Cell Mol Biol Lett 25: 1, 2020.

11. Jiang Q, Li F, Cheng Z, Kong Y and Chen C: The role of E3 ubiquitin ligase HECTD3 in cancer and beyond. Cell Mol Life Sci 77: $1483-1495,2020$

12. Liu X, Zurlo G and Zhang Q: The roles of Cullin-2 E3 ubiquitin ligase complex in cancer. Adv Exp Med Biol 1217: 173-186, 2020.

13. Venuto S and Merla G: E3 ubiquitin ligase TRIM proteins, cell cycle and mitosis. Cells 8: 510, 2019.

14. Wang ZW, Hu X, Ye M, Lin M, Chu M and Shen X: NEDD4 E3 ligase: Functions and mechanism in human cancer. Semin Cancer Biol 67: 92-101, 2020.

15. Katoh $\mathrm{M}$ and Katoh $\mathrm{M}$ : Identification and characterization of PDZRN3 and PDZRN4 genes in silico. Int J Mol Med 13: 607-613, 2004

16. Ko JA, Kimura Y, Matsuura K, Yamamoto H, Gondo T and Inui M: PDZRN3 (LNX3, SEMCAP3) is required for the differentiation of C2C12 myoblasts into myotubes. J Cell Sci 119: 5106-5113, 2006.

17. Marracci S, Vangelisti A, Raffa V, Andreazzoli M and Dente L: pdzrn3 is required for pronephros morphogenesis in Xenopus laevis. Int J Dev Biol 60: 57-63, 2016.

18. Marunaka K, Furukawa C, Fujii N, Kimura T, Furuta T, Matsunaga T, Endo S, Hasegawa H, Anzai N, Yamazaki Y, et al: The RING finger- and PDZ domain-containing protein PDZRN3 controls localization of the $\mathrm{Mg}^{2+}$ regulator claudin-16 in renal tube epithelial cells. J Biol Chem 292: 13034-13044, 2017.

19. Livak KJ and Schmittgen TD: Analysis of relative gene expression data using real-time quantitative PCR and the 2(-Delta Delta C(T)) method. Methods 25: 402-408, 2001

20. Sewduth RN, Jaspard-Vinassa B, Peghaire C, Guillabert A, Franzl N, Larrieu-Lahargue F, Moreau C, Fruttiger M, Dufourcq $\mathrm{P}$, Couffinhal $\mathrm{T}$ and Duplàa $\mathrm{C}$ : The ubiquitin ligase PDZRN3 is required for vascular morphogenesis through Wnt/planar cell polarity signaling. Nat Commun 5: 4832, 2014.

21. Hanahan D and Weinberg RA: Hallmarks of cancer: The next generation. Cell 144: 646-674, 2011.

22. Nikolaou S and Machesky LM: The stressful tumour environment drives plasticity of cell migration programmes, contributing to metastasis. J Pathol 250: 612-623, 2020.

23. Jamil A and Kasi A: Lung Metastasis. In: StatPearls (Internet). StatPearls Publishing, Treasure Island, FL, 2021.

24. Lu C, Ning G, Si P, Zhang C, Liu W, Ge W, Cui K, Zhang R and Ge S: E3 ubiquitin ligase HECW1 promotes the metastasis of non-small cell lung cancer cells through mediating the ubiquitination of Smad4. Biochem Cell Biol 99: 675-681, 2021.

25. Shen J, Yu Z and Li N: The E3 ubiquitin ligase RNF146 promotes colorectal cancer by activating the $\mathrm{Wnt} / \beta$-catenin pathway via ubiquitination of Axin1. Biochem Biophys Res Commun 503: 991-997, 2018.

26. Honda $\mathrm{T}$ and Inui M: PDZRN3 regulates differentiation of myoblasts into myotubes through transcriptional and posttranslational control of Id2. J Cell Physiol 234: 2963-2972, 2019.

27. Honda T and Inui M: PDZRN3 protects against apoptosis in myoblasts by maintaining cyclin A2 expression. Sci Rep 10: $1140,2020$.

28. Sewduth RN, Kovacic H, Jaspard-Vinassa B, Jecko V, Wavasseur T, Fritsch N, Pernot M, Jeaningros S, Roux E, Dufourcq P, et al: PDZRN3 destabilizes endothelial cell-cell junctions through a PKC $\zeta$-containing polarity complex to increase vascular permeability. Sci Signal 10: eaag3209, 2017.

29. Chatterjee A,Paul S, Bisht B, Bhattacharya S, Sivasubramaniam S and Paul MK: Advances in targeting the $\mathrm{WNT} / \beta$-catenin signaling pathway in cancer. Drug Discov Today: Jul 10, 2021 (Epub ahead of print).

30. Fatima I, Barman S, Rai R, Thiel KWW and Chandra V: Targeting Wnt signaling in endometrial cancer. Cancers (Basel) 13: 2351, 2021. 